\title{
Rare case of bilateral massive adrenal myelolipoma in association with congenital adrenal hyperplasia
}

Dr.Shahbuddin.M.1 ${ }^{1}$, Dr.David Hughes ${ }^{1}$, Dr. Efthymios Hadjimichael ${ }^{2}$,Mr. Simon Williams ${ }^{3}$

${ }^{1}$ Department of Endocrinology, ${ }^{2}$ Department of Histopathology, ${ }^{3}$ Department of Urosurgery, Royal Derby Hospital, Derby

Introduction: Adrenal myelolipomas are rare small benign tumours composed of mature fat and hemopoietic tissues, which can be associated with congenital adrenal hyperplasia (CAH). We report a rare case of massive bilateral adrenal myelolipomas.

Case report: A 48 year old gentleman with CAH diagnosed during childhood presented at a routine endocrine appointment after missing several appointments and steroids for over seven years. Clinical examination revealed bilateral enlarged testicles along with a palpable abdominal mass.

Imaging: Coincidentally he had undergone an abdominal ultrasound organised by his primary care physician for suspected cholecystitis. The scan revealed bilateral abdominal masses which was further characterised by CT/MRI abdomen as bilateral massive adrenal myelolipomas displacing abdominal viscera.

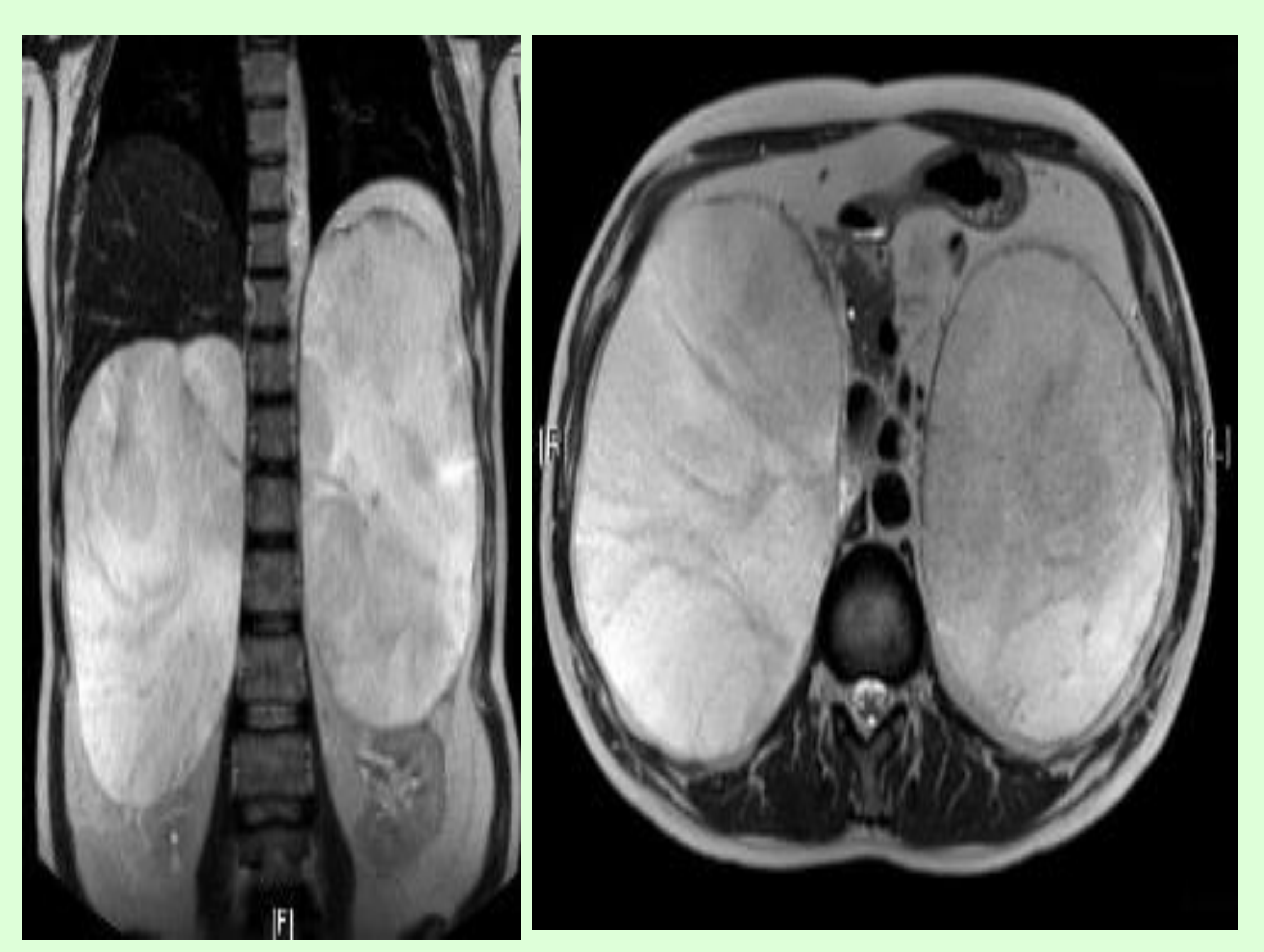

MRI Abdomen : Right adrenal measuring $20 \mathrm{~cm}$ \& Left adrenal, $18 \mathrm{~cm}$.

Management: Initiation of steroid therapy resulted in testicular shrinkage, erectile dysfunction and decline in early morning testosterone from $11 \mathrm{nmol} / \mathrm{l}$ to $0.3 \mathrm{nmol} / \mathrm{I}$ [normal=8.3$27.8 \mathrm{nmol} / \mathrm{l}$.

Surgery: He underwent successful elective bilateral adrenalectomy and made a good post operative recovery. He was discharged home on Prednisolone, Fludrocortisone and testosterone replacement.

Genetic Screening: This showed CYP21A2 abnormality in a heterozygous conversion, a known mutation resulting in CAH due to 21-hydroxylalse deficiency.

\section{Macroscopy and Microscopy}

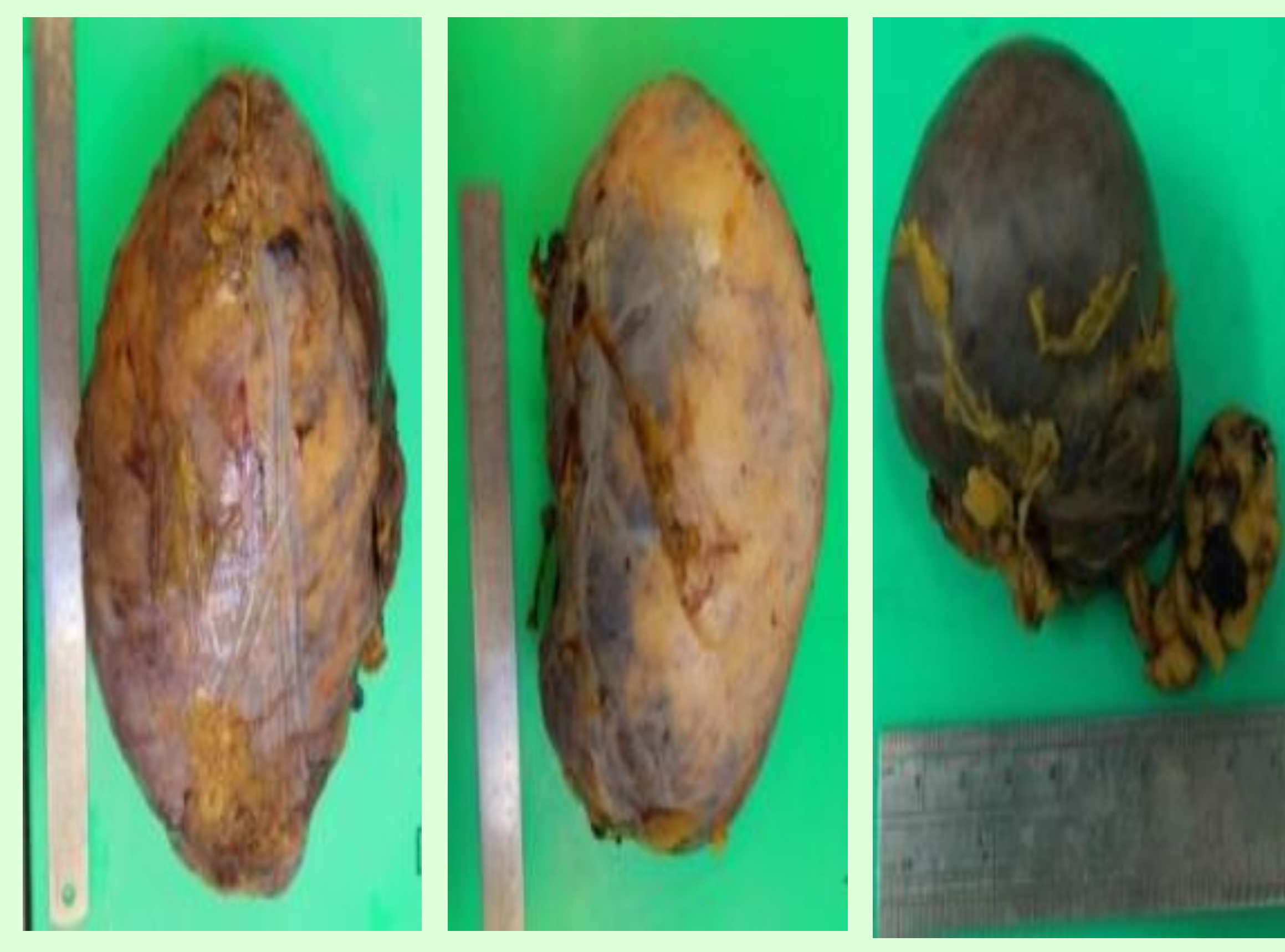

Right-2.9kg Left-2.1 kg Left Adrenunculus-

$27 \times 21 \mathrm{cms} \quad 25 \times 15 \mathrm{cms} \quad 85 \mathrm{gms}$

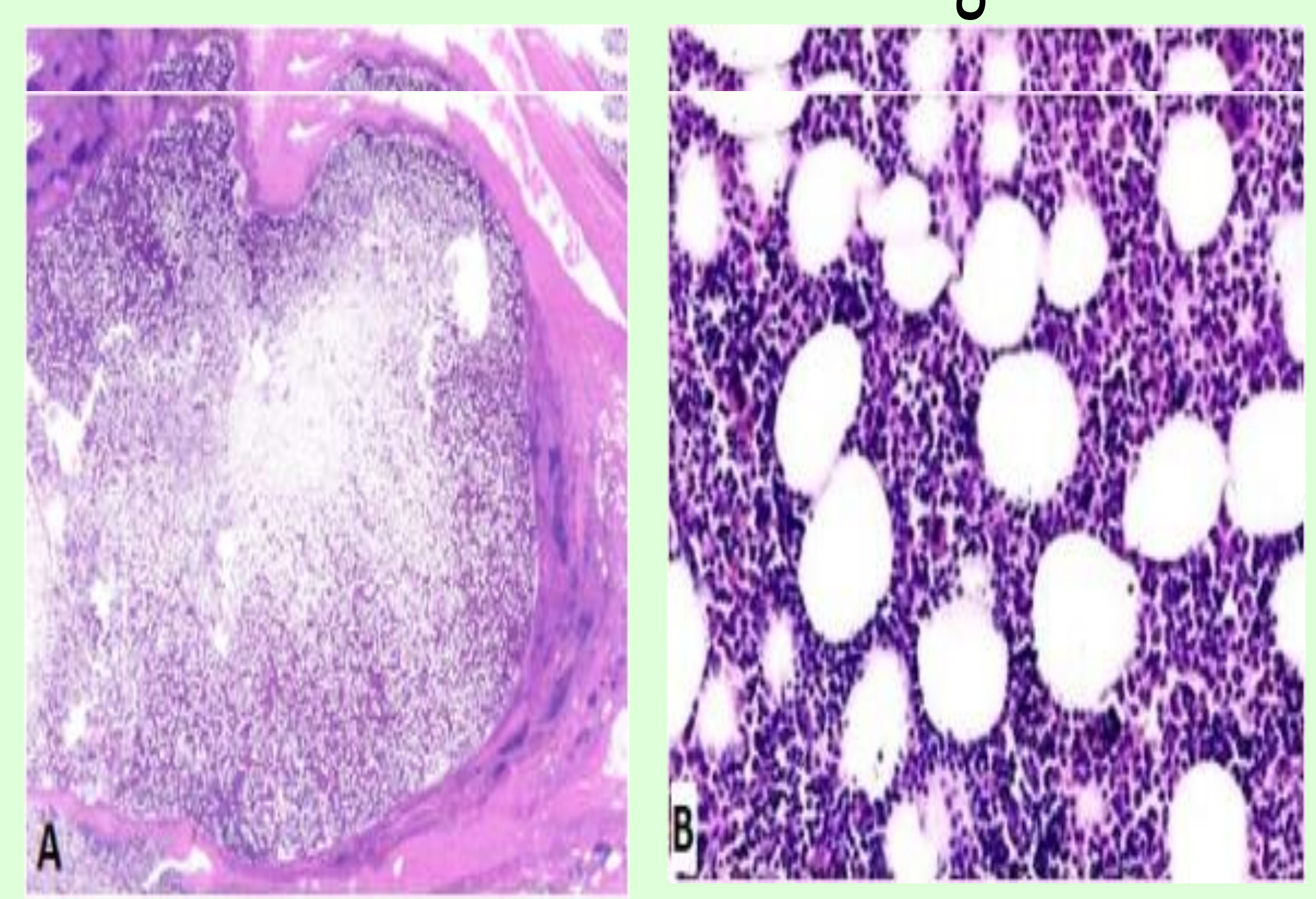

Histology (A) shows a fairly well circumscribed lesion consisting of mature fatty tissue with scattered haematopoietic elements. On higher magnification (X20) (B) the lesion shows full trilineage maturation of myeloid, erythroid and megakaryocytic cell lines Discussion: Etiopathogenesis of adrenal myelolipomas still remains unclear in spite of various theories. Being off steroids in the presence of CAH was probably the causative factor in our patient. Small and asymptomatic tumours can be managed conservatively with annual follow up but lesions more than $5 \mathrm{cms}$ and the symptomatic tumours usually require surgical resection. Several cases of unilateral massive adrenal myelolipomas have been reported but bilateral massive adrenal myelolipomas are extremely rare.

References:

Wani N., Kosar T., Rawa I., Qayum A. Giant adrenal myelolipoma: incidentaloma with a rare incidental association. Urol Ann. 2010;2.3:130

Al-Bahri, S. et al. "Giant Bilateral Adrenal Myelolipoma with Congenital Adrenal Hyperplasia." Case Reports in Surgery 2014 (2014): 728198. 